\title{
Red meat consumption is associated with the risk of type 2 diabetes in men but not in women: a Japan Public Health Center-based Prospective Study
}

\author{
Kayo Kurotani $^{1 *}$, Akiko Nanri ${ }^{1}$, Atsushi Goto ${ }^{2}$, Tetsuya Mizoue ${ }^{1}$, Mitsuhiko Noda $^{2}$, Shino Oba ${ }^{3}$, \\ Masayuki Kato $^{4}$, Yumi Matsushita ${ }^{5}$, Manami Inoue ${ }^{6}$ and Shoichiro Tsugane ${ }^{6}$ for the Japan Public \\ Health Center-based Prospective Study Group \\ ${ }^{1}$ Department of Epidemiology and Prevention, Clinical Research Center, National Center for Global Health \\ and Medicine, Tokyo 162-8655, Japan \\ ${ }^{2}$ Department of Diabetes and Metabolic Medicine, National Center for Global Health and Medicine, Tokyo, Japan \\ ${ }^{3}$ Department of Health Promotion, National Institute of Public Health, Saitama, Japan \\ ${ }_{4}^{4}$ Japan Foundation for the Promotion of International Medical Research Cooperation, Tokyo, Japan \\ ${ }^{5}$ Department of Clinical Research Coordination, National Center for Global Health and Medicine, Tokyo, Japan \\ ${ }^{6}$ Epidemiology and Prevention Division, Research Center for Cancer Prevention and Screening, \\ National Cancer Center, Tokyo, Japan
}

(Submitted 30 August 2012 - Final revision received 8 March 2013 - Accepted 16 March 2013 - First published online 7 May 2013)

\section{Abstract}

The relationship between different types of meat intake and the risk of type 2 diabetes remains unclear. We prospectively examined the association between total meat, total red meat, unprocessed red meat, processed meat and poultry intake and the incidence of type 2 diabetes. Subjects were 27425 men and 36424 women aged 45-75 years who participated in the second survey of the Japan Public Health Center-based Prospective Study, and had no history of type 2 diabetes, cancer, stroke, IHD, chronic liver disease or kidney disease. Meat intake was estimated using a validated 147-item FFQ. OR of self-reported, physician-diagnosed type 2 diabetes over 5 years were estimated using a multiple logistic regression. A total of 1178 newly diagnosed cases of type 2 diabetes were self-reported. Intakes of total meat and total red meat were associated with the increased risk of type 2 diabetes in men but not in women. The multivariate-adjusted OR for the highest quartile compared with the lowest quartile of total meat and total red meat intake were 1.36 (95\% CI $1.07,1.73 ; P$ for trend $=0.006)$ and $1.48(95 \%$ CI $1.15,1.90 ; P$ for trend=0.003) for men, respectively, and $0.82(95 \%$ CI $0.62,1.09 ; P$ for trend $=0.14)$ and $0.77(95 \%$ CI $0.57,1.02 ; P$ for trend=0.08) for women, respectively. Intakes of processed red meat and poultry were not associated with the increased risk of diabetes in either men or women. In conclusion, elevated intake of red meat is associated with the increased risk of type 2 diabetes in Japanese men but not in women.

Key words: Meats: Red meat: Processed meats: Poultry: Type 2 diabetes

The incidence of diabetes has been increasing globally, with an estimated world prevalence reaching $7 \cdot 7 \%$ by $2030^{(1)}$. In Japan, the prevalence of diabetes has increased markedly over the last few decades ${ }^{(2)}$, and it has been argued that the increase is primarily due to the Westernisation of diet, including the increased intake of animal products ${ }^{(3)}$. Data from prospective studies have indicated that total meat intake is consistently associated with the increased risk of type 2 diabetes among men ${ }^{(4-6)}$, whereas inconsistent findings have been observed in women ${ }^{(4,7)}$. The results of recent meta-analyses of prospective studies support that a high red meat ${ }^{(8,9)}$ and processed meat ${ }^{(8-10)}$ intake increases the risk of type 2 diabetes in both men and women. $\mathrm{Fe}$, which is contained in red meat, may play a role as a mediator of the adverse effect of red meat intake on glucose metabolism $^{(11,12)}$. Additionally, SFA in meat may increase inflammatory response and secondarily enhance the risk of type 2 diabetes $^{(13)}$. In terms of poultry intake, cohort studies have reported no association among men ${ }^{(4-6,14,15)}$, but data among women are conflicting ${ }^{(4,7,14-16)}$.

Epidemiological evidence on this issue is limited among Asian populations, in which meat consumption is much lower than that in Western populations ${ }^{(17)}$. In a study of Chinese women, the only prospective investigation in Asia, intake of total meat

Abbreviation: JPHC, Japan Public Health Center-based Prospective.

*Corresponding author: Dr K. Kurotani, fax +81 33202 7364, email kkurotani@ri.ncgm.go.jp 
(red meat and poultry), was inversely associated with the risk of type 2 diabetes ${ }^{(7)}$, a finding that conflicts with the findings of Western studies ${ }^{(4-6,18)}$, although the association between processed meat intake and the risk of type 2 diabetes in the Chinese study ${ }^{(7)}$ is consistent with those in Western studies $^{(4,5,14-16,19-23)}$. Japanese patients with type 2 diabetes are on average leaner than their Western counterparts ${ }^{(24)}$, and Japanese-Americans have lower $\beta$-cell function than do non-Hispanic whites ${ }^{(25)}$. Thus, the effect of meat consumption on type 2 diabetes in the Japanese population may differ from the effect in Western populations. Here, we prospectively investigated the association between meat intake (total red meat, unprocessed red meat, processed red meat and poultry) and the risk of type 2 diabetes in a large-scale, populationbased cohort of Japanese men and women. Further, we examined the association by BMI, which is a major predictor of type 2 diabetes risk, and by menopausal status (women only), which is a determinant of body Fe storage that may increase with the intake of Fe-containing foods including meat, and which has been linked to type 2 diabetes risk ${ }^{(26)}$.

\section{Materials and methods}

\section{Study design}

Cohort I of the Japan Public Health Center-based Prospective (JPHC) Study was established in 1990, and cohort II was established in $1993^{(27)}$. The study protocol was approved by the institutional review board of the National Cancer Center, Tokyo, Japan. The participants of cohort I included residents aged 40-59 years in five Japanese public health centre areas (Iwate, Akita, Nagano, Okinawa and Tokyo). The participants of cohort II included residents aged 40-69 years in six public health centre areas (Ibaraki, Niigata, Kochi, Nagasaki, Okinawa and Osaka). Although we did not require written informed consent, the study participants were informed of the objectives of the study, and participants who responded to the questionnaire survey were considered to have consented to participate in the survey.

Among the baseline subjects ( $n$ 140 420), 113403 subjects responded to the questionnaire survey at baseline. Of these, $89947(79 \cdot 3 \%)$ subjects responded to the 5-year follow-up survey (second survey). Of these subjects, 76901 (67.8\%) responded to the 10-year follow-up survey (third survey). We excluded 12462 subjects who reported a history of type 2 diabetes, cancer, stroke, IHD and chronic liver disease at baseline or at the second survey, as well as those who reported kidney disease at the baseline survey. Individuals who were missing information regarding their meat intake were excluded. We also excluded 590 subjects who reported extreme total energy intakes (outside of the mean $\pm 3 \mathrm{SD}$ according to sex). Finally, a total of 63849 subjects (27 425 men and 36424 women) remained in the present analysis.

\section{$F F Q$}

Participants completed a self-administered FFQ at the baseline, second and third surveys. The data included 147 food and beverage items and nine frequency categories ${ }^{(28)}$. For the present analysis, we used data from the second survey as the baseline data because the questionnaire used for the second survey more comprehensively inquired about food intakes than the one used for the baseline survey. At the second survey, we asked about the usual consumption of sixteen meat items over the past year ${ }^{(28)}$. The red meat items included three beef dishes (steak, grilled beef and stewed beef), six pork dishes (stir-fried pork, deep-fried pork, stewed pork in the Western style, stewed pork in the Japanese style, pork in soup and pork liver), four processed meat products (ham, sausage or Wiener sausage, bacon and luncheon meat) and chicken liver. Poultry items included two chicken meals (grilled chicken and deep-fried chicken). For most food items, nine response options were available to describe consumption frequency, ranging from rarely ( $<1$ time/month) to $\geq 7$ times/d. A standard portion size was specified for each food, and respondents were asked to choose their usual portion size from three options $(\leq 0.5$ times, standard or $\geq 1.5$ times). The daily intake of meat and meat products was calculated by multiplying the daily consumption frequency by the typical portion size, and was expressed as $\mathrm{g} / \mathrm{d}$. These individual items were categorised into five main groups of total meat, total red meat (unprocessed and processed red meat items), unprocessed red meat, processed red meat and poultry.

Referring to the Standard Tables of Food Composition in Japan ${ }^{(29)}$, dietary intakes for energy and selected nutrients were estimated. The validity and reproducibility of the FFQ was examined in a subsample of the participants in the JPHC Study cohort I and cohort II. Details of the validation study have been described elsewhere ${ }^{(30-32)}$. For the validity of the FFQ, energy-adjusted Spearman's correlation COefficients between intake values for meat derived from the FFQ and those derived from 28 or $14 \mathrm{~d}$ dietary records were 0.50 for men and 0.45 for women, respectively, for cohort $\mathrm{I}^{(32)}$, and 0.48 for men and 0.44 for women, respectively, for cohort $\mathrm{II}^{(30)}$. With regard to the reproducibility of the FFQ, energy-adjusted Spearman's correlation coefficients for intake of meat derived from the two FFQ administered 1 year apart were 0.52 for both men and women for cohort $\mathrm{I}^{(31)}$ and 0.52 for men and 0.41 for women, respectively, for cohort $\mathrm{II}^{(30)}$.

\section{Ascertainment of type 2 diabetes}

Type 2 diabetes was ascertained using a self-administered questionnaire. At the third survey, study participants were asked about their history of major diseases including diabetes and, if it was present, the timing of the initial diagnosis in relation to the first and second surveys. Because the 5-year survey was used as the baseline measure in the present study, only participants who were subsequently diagnosed were regarded as incident cases during the follow-up. Details regarding the assessment of the validity of self-reported diabetes have been described elsewhere ${ }^{(33)}$. Previously, we showed that $94 \%$ of self-reported diabetes cases were confirmed by medical records. 


\section{Statistical analyses}

Analyses of men and women were performed separately. Participants were divided into intake quartiles. The confounding variables considered were as follows: age (years, continuous); study area (eleven areas); BMI ( $<21,21-22 \cdot 9,23-24 \cdot 9$, $25-26.9$ or $\geq 27 \mathrm{~kg} / \mathrm{m}^{2}$ ); smoking status (lifetime non-smoker, former smoker or current smoker with a consumption of either $<20$ or $\geq 20$ cigarettes/d); alcohol consumption (nondrinker, occasional drinker or drinker with a consumption of $<150$, 150-299, 300-499 or $\geq 450 \mathrm{~g}$ ethanol/week for men and $<150$ or $\geq 150 \mathrm{~g}$ ethanol/week for women); total physical activity level (metabolic equivalent-h/d, quartiles); history of hypertension (yes or no); family history of diabetes mellitus (yes or no); coffee consumption (almost never, $<1,1$ or $\geq 2$ cups/d); total energy intake (kJ/d, continuous); Ca intake ( $\mathrm{mg} / \mathrm{d}$, continuous); $\mathrm{Mg}$ intake ( $\mathrm{mg} / \mathrm{d}$, continuous); rice intake (g/d, continuous); fish intake (g/d, continuous); vegetable intake $(\mathrm{g} / \mathrm{d}$, continuous); soft drink intake $(\mathrm{g} / \mathrm{d}$, continuous). The dietary factors considered here have been shown to be associated with the risk of type 2 diabetes both in previous studies and in the present cohort. An indicator variable for missing data was created for each covariate. Trend associations between confounding factors and meat intakes were examined using the Mantel-Haenszel $\chi^{2}$ test for categorical variables and linear regression analysis for continuous variables.

The association between the intakes of energy-adjusted total meat, total red meat, unprocessed red meat, processed red meat or poultry and the risk of diabetes was assessed by OR, which were estimated using a multiple logistic regression. A $95 \%$ CI of the OR was estimated using the Wald method. The first model was adjusted for age and study area, and the second was further adjusted for BMI, smoking status, alcohol consumption, the family history of diabetes mellitus, the history of hypertension, total physical activity level, total energy intake, coffee consumption and the intakes of $\mathrm{Ca}$, $\mathrm{Mg}$, rice, fish, vegetables and soft drinks. An additional model was further adjusted for Fe intake ( $\mathrm{g} / \mathrm{d}$, continuous) or saturated fat intake ( $\mathrm{g} / \mathrm{d}$, continuous). The trend association was assessed by assigning the ordinal numbers $0-3$ to the four categories of each meat or specific groups of meat consumption. We also analysed data by BMI $\left(<25\right.$ or $\left.\geq 25 \mathrm{~kg} / \mathrm{m}^{2}\right)$ in both men and women and menopausal status (pre-menopausal or postmenopausal) in women. An interaction term of dietary intake (continuous) and the above stratifying variables (dichotomous) was created and added to the model to assess statistical interactions. Statistical significance was declared if the two-sided $P$ value was less than 0.05. All analyses were performed using SAS software (version 9.2; SAS Institute).

\section{Results}

During the 5-year period, 1178 participants (681 men and 497 women) were newly self-reported as having type 2 diabetes. At baseline (the time of the second survey), both men and women with higher intakes of total meat were more likely to be young and to have a high BMI. They also had higher intakes of protein, fat, soft drinks and coffee, and lower intakes of carbohydrates, $\mathrm{Ca}, \mathrm{Mg}$, rice and vegetable than those with lower total meat intakes (Table 1). Men who consumed greater amounts of total meat were less likely to report higher levels of physical activity at work or during leisure time and to consume alcohol.

Total meat was positively associated with the risk of type 2 diabetes in men (Table 2). The OR in the highest quartile of total meat intake compared with those in the lowest was 1.36 (95\% CI $1.07,1.73$; $P$ for trend=0.006) in the multivariate-adjusted model. The median intake of total red meat was 45.6 and $40.1 \mathrm{~g} / \mathrm{d}$ in men and women, respectively. High intakes of total red meat and unprocessed red meat were statistically significantly associated with an increased risk of type 2 diabetes in men. The multivariate-adjusted OR of type 2 diabetes for the lowest to the highest quartile category of intake were 1.00 (reference), 1.09 (95\% CI 0.87, 1.37), 1.15 (95\% CI $0.91,1.46)$ and 1.48 (95\% CI $1.15,1.90)$ ( $P$ for trend $=0.003$ ) and 1.00 (reference), 0.99 (95\% CI 0.79, 1.24), 1.01 (95\% CI $0.80,1.27)$ and $1.42(95 \%$ CI $1.12,1.80)(P$ for trend $=0.007)$ for total red meat and unprocessed red meat, respectively. These associations were only slightly attenuated after additional adjustment for Fe intake. We did not observe significant associations of total red meat and unprocessed red meat with the risk of type 2 diabetes in women. Poultry consumption was not associated with the risk of type 2 diabetes among either men or women. In stratified analyses, there was no significant interaction by BMI or menopausal status (women only).

\section{Discussion}

In the present large-scale population-based prospective study in Japanese adults, a high consumption of total meat, total red meat and unprocessed red meat were associated with the increased risk of type 2 diabetes in men but not in women. Processed red meat and poultry intakes were not associated with an increased risk of diabetes in men or women. Thus, the associations with total meat and total red meat are largely accounted for by the association with unprocessed red meat.

The present finding of a positive association of total meat intake with the risk of type 2 diabetes in men is consistent with a recent meta-analysis of three cohort studies from the USA; the summary relative risk of type 2 diabetes per $100 \mathrm{~g} / \mathrm{d}$ of total meat was 1.12 (95\% CI $1.05,1 \cdot 19)^{(10)}$. Although another meta-analysis of five prospective studies from the USA, Australia, Japan and China reported no clear association of total meat intake with the risk of type 2 diabetes, a statistically significantly increased risk associated with a high intake of total meat emerged after excluding a Chinese study ${ }^{(7)}$; the summary relative risk of type 2 diabetes comparing a high $v$. low intake of total meat was $1.31(95 \% \mathrm{CI}$ $1 \cdot 12,1.52)^{(8)}$. In contrast, the present study found no association in women, a finding that is inconsistent with those found for women in a Western study ${ }^{(4)}$. The above-mentioned study in Chinese women reported a decreased, rather than increased, risk of type 2 diabetes associated with a higher intake of total meat. Although caution needs to be exercised 
Table 1. Baseline characteristics of the subjects according to categories of energy-adjusted meat intake*

(Mean values and standard deviations; percentages)

\begin{tabular}{|c|c|c|c|c|c|c|c|c|}
\hline & \multicolumn{8}{|c|}{ Quartiles of total meat intake } \\
\hline & \multicolumn{2}{|c|}{ Q1 } & \multicolumn{2}{|c|}{ Q2 } & \multicolumn{2}{|c|}{ Q3 } & \multicolumn{2}{|c|}{ Q4 } \\
\hline & Mean & SD & Mean & SD & Mean & SD & Mean & SD \\
\hline \multicolumn{9}{|l|}{ Men } \\
\hline$n$ & \multicolumn{2}{|c|}{6856} & \multicolumn{2}{|c|}{6856} & \multicolumn{2}{|c|}{6857} & \multicolumn{2}{|c|}{6856} \\
\hline Age (years)† & $52 \cdot 2$ & $7 \cdot 5$ & $51 \cdot 0$ & $7 \cdot 7$ & $50 \cdot 5$ & $7 \cdot 7$ & $50 \cdot 7$ & $7 \cdot 8$ \\
\hline BMI $\left(\mathrm{kg} / \mathrm{m}^{2}\right) \dagger$ & $23 \cdot 4$ & $2 \cdot 7$ & $23 \cdot 5$ & $2 \cdot 8$ & $23 \cdot 6$ & $2 \cdot 8$ & $23 \cdot 8$ & 2.9 \\
\hline Current smoker $(\%) \dagger$ & \multicolumn{2}{|c|}{44.5} & \multicolumn{2}{|c|}{$46 \cdot 9$} & \multicolumn{2}{|c|}{$46 \cdot 0$} & \multicolumn{2}{|c|}{$44 \cdot 0$} \\
\hline Alcohol consumption $\geq 1 \mathrm{~d} /$ week $(\%) \dagger$ & \multicolumn{2}{|c|}{$71 \cdot 3$} & \multicolumn{2}{|c|}{$71 \cdot 2$} & \multicolumn{2}{|c|}{$68 \cdot 6$} & \multicolumn{2}{|c|}{$58 \cdot 5$} \\
\hline Total physical activity (MET-h/d)† & $34 \cdot 2$ & $6 \cdot 8$ & $34 \cdot 1$ & $6 \cdot 8$ & $33 \cdot 8$ & $6 \cdot 7$ & 33.7 & $6 \cdot 7$ \\
\hline History of hypertension (\%)† & & & & & & & & \\
\hline Family history of diabetes (\%) & & & & & & & & \\
\hline Food and nutrient intake $\ddagger$ & & & & & & & & \\
\hline Energy $(\mathrm{kJ} / \mathrm{d}) \dagger$ & 9532 & 3156 & 9473 & 2901 & 9385 & 2951 & 9146 & 3282 \\
\hline Protein $(g / d) \dagger$ & 68 & 15 & 71 & 13 & 73 & 12 & 77 & 12 \\
\hline Carbohydrate $(\mathrm{g} / \mathrm{d}) \dagger$ & 300 & 53 & 291 & 45 & 279 & 42 & 251 & 43 \\
\hline Fat $(g / d) \dagger$ & 44 & 14 & 50 & 12 & 56 & 11 & 70 & 15 \\
\hline Saturated fat $(\mathrm{g} / \mathrm{d}) \dagger$ & 13 & 6 & 15 & 5 & 17 & 4 & 21 & 5 \\
\hline Monounsaturated fat $(\mathrm{g} / \mathrm{d}) \dagger$ & 14 & 4 & 17 & 4 & 20 & 4 & 26 & 6 \\
\hline Polyunsaturated fat $(\mathrm{g} / \mathrm{d}) \dagger$ & 11 & 4 & 12 & 3 & 13 & 3 & 15 & 3 \\
\hline $\mathrm{Ca}(\mathrm{mg} / \mathrm{d}) \dagger$ & 543 & 274 & 512 & 216 & 486 & 192 & 448 & 179 \\
\hline $\mathrm{Mg}(\mathrm{mg} / \mathrm{d}) \dagger$ & 288 & 63 & 283 & 53 & 278 & 49 & 265 & 50 \\
\hline $\mathrm{Fe}(\mathrm{mg} / \mathrm{d})$ & $9 \cdot 1$ & 2.5 & $9 \cdot 0$ & $2 \cdot 1$ & $9 \cdot 0$ & $2 \cdot 1$ & $9 \cdot 1$ & $2 \cdot 2$ \\
\hline Rice $(g / d) \dagger$ & 470 & 195 & 455 & 172 & 430 & 155 & 374 & 146 \\
\hline Vegetables $(g / d) \dagger$ & 198 & 146 & 197 & 121 & 194 & 119 & 190 & 115 \\
\hline Fish $(g / d) \dagger$ & 87 & 61 & 90 & 51 & 93 & 49 & 92 & 52 \\
\hline Soft drinks $(g / d) \dagger$ & 42 & 242 & 49 & 195 & 59 & 220 & 90 & 326 \\
\hline Coffee consumption $\geq 1 \mathrm{cup} / \mathrm{d}(\%) \dagger$ & & & & & & & & \\
\hline Women & & & & & & & & \\
\hline$n$ & & & & & & & & \\
\hline Age (years)† & $52 \cdot 6$ & $7 \cdot 7$ & $51 \cdot 5$ & $7 \cdot 7$ & $51 \cdot 0$ & $7 \cdot 8$ & $51 \cdot 1$ & $8 \cdot 0$ \\
\hline BMI $\left(\mathrm{kg} / \mathrm{m}^{2}\right) \dagger$ & $23 \cdot 4$ & $3 \cdot 1$ & $23 \cdot 4$ & $3 \cdot 0$ & 23.4 & $3 \cdot 1$ & $23 \cdot 7$ & $3 \cdot 2$ \\
\hline Current smoker $(\%) \dagger$ & & & & & & & & \\
\hline Alcohol consumption $\geq 1 \mathrm{~d} /$ week $(\%)$ & & & & & & & & \\
\hline Total physical activity (MET-h/d) & 32.9 & $5 \cdot 7$ & $32 \cdot 9$ & $5 \cdot 7$ & $32 \cdot 9$ & 5.7 & $32 \cdot 8$ & $5 \cdot 8$ \\
\hline History of hypertension (\%)† & & & & & & & & \\
\hline Family history of diabetes (\%)† & & & & & & & & \\
\hline Postmenopausal status (\%)† & & & & & & & & \\
\hline Food and nutrient intake $\neq$ & & & & & & & & \\
\hline Energy $(\mathrm{kJ} / \mathrm{d}) \dagger$ & 8251 & 2972 & 8092 & 2620 & 8037 & 2541 & 7790 & 2754 \\
\hline Protein $(g / d) \dagger$ & 67 & 11 & 68 & 10 & 69 & 9 & 71 & 10 \\
\hline Carbohydrate $(\mathrm{g} / \mathrm{d}) \dagger$ & 275 & 35 & 263 & 29 & 250 & 26 & 224 & 32 \\
\hline Fat $(g / d) \dagger$ & 47 & 11 & 52 & 10 & 56 & 9 & 67 & 13 \\
\hline Saturated fat $(\mathrm{g} / \mathrm{d}) \dagger$ & 14 & 5 & 16 & 4 & 17 & 4 & 20 & 5 \\
\hline Monounsaturated fat $(\mathrm{g} / \mathrm{d}) \dagger$ & 15 & 4 & 17 & 3 & 19 & 3 & 24 & 5 \\
\hline Polyunsaturated fat $(\mathrm{g} / \mathrm{d}) \dagger$ & 12 & 3 & 12 & 3 & 13 & 3 & 14 & 3 \\
\hline $\mathrm{Ca}(\mathrm{mg} / \mathrm{d}) \dagger$ & 616 & 254 & 571 & 201 & 530 & 177 & 468 & 179 \\
\hline $\mathrm{Mg}(\mathrm{mg} / \mathrm{d}) \dagger$ & 289 & 55 & 277 & 45 & 268 & 42 & 250 & 47 \\
\hline $\mathrm{Fe}(\mathrm{mg} / \mathrm{d}) \dagger$ & $9 \cdot 2$ & 2.3 & $9 \cdot 0$ & 1.9 & 8.9 & 1.9 & 8.7 & $2 \cdot 0$ \\
\hline Rice $(g / d) \dagger$ & 361 & 153 & 354 & 133 & 346 & 126 & 313 & 122 \\
\hline Vegetables $(g / d) \dagger$ & 249 & 159 & 236 & 126 & 222 & 115 & 206 & 119 \\
\hline Fish $(g / d)$ & 85 & 55 & 86 & 46 & 88 & 43 & 85 & 47 \\
\hline Soft drinks $(g / d) \dagger$ & 12 & 56 & 15 & 67 & 17 & 66 & 26 & 95 \\
\hline Coffee consumption $\geq 1 \mathrm{cup} / \mathrm{d}(\%) \dagger$ & & & & & & & & \\
\hline
\end{tabular}

MET, metabolic equivalents.

* On the basis of the Mantel-Haenszel $\chi^{2}$ test for categorical variables and linear regression analysis for continuous variables with the assignment of ordinal numbers $0-3$ to the categories of total meat intake.

$\dagger P$ for trend $<0.05$.

$\ddagger$ Energy adjusted by the residual method except for energy intake and coffee consumption.

when interpreting the results of the Chinese study, as their measure of total meat included unprocessed red meat and poultry but not processed meat ${ }^{(7)}$, available evidence from Japan and China did not support the hypothesis that total meat intake is associated with the increased risk of type 2 diabetes among female Asian populations.

The present finding of a positive association with the consumption of total red meat, which is mainly derived from 
Table 2. Type 2 diabetes according to the quartile categories of energy-adjusted meat intakes

(Odds ratios and 95\% confidence intervals)

\begin{tabular}{|c|c|c|c|c|c|c|c|c|}
\hline & \multicolumn{7}{|c|}{ Quartile category } & \multirow[b]{3}{*}{$P$ for trend* } \\
\hline & \multirow{2}{*}{$\begin{array}{l}\text { Q1 } \\
\text { OR }\end{array}$} & \multicolumn{2}{|c|}{ Q2 } & \multicolumn{2}{|c|}{ Q3 } & \multicolumn{2}{|c|}{ Q4 } & \\
\hline & & OR & $95 \% \mathrm{Cl}$ & OR & $95 \% \mathrm{Cl}$ & OR & $95 \% \mathrm{Cl}$ & \\
\hline \multicolumn{9}{|l|}{ Men } \\
\hline \multicolumn{9}{|l|}{ Total meat } \\
\hline Cases/subjects $(n)$ & $166 / 6856$ & \multicolumn{2}{|c|}{$143 / 6856$} & \multicolumn{2}{|c|}{$168 / 6857$} & \multicolumn{2}{|c|}{$204 / 6856$} & \\
\hline Median $(\mathrm{g})$ & $23 \cdot 2$ & & $44 \cdot 8$ & & $7 \cdot 2$ & & $07 \cdot 8$ & \\
\hline Age- and area-adjusted OR $\dagger$ & 1.00 (reference) & 0.87 & $0.69,1.09$ & 1.03 & $0.83,1.29$ & 1.26 & $1.02,1.57$ & 0.01 \\
\hline Multivariate-adjusted OR $\ddagger$ & 1.00 (reference) & 0.88 & $0.70,1 \cdot 11$ & 1.06 & $0.85,1.34$ & 1.36 & $1.07,1.73$ & 0.006 \\
\hline Plus Fe-adjusted OR $\ddagger$ & 1.00 (reference) & 0.88 & $0.70,1 \cdot 11$ & 1.06 & $0.84,1.33$ & 1.33 & $1.05,1.70$ & 0.01 \\
\hline Plus saturated fat-adjusted OR & 1.00 (reference) & 0.89 & $0.71,1.13$ & $1 \cdot 10$ & $0.85,1.41$ & 1.44 & $1.05,1.99$ & 0.03 \\
\hline Total red meat & & & & & & & & \\
\hline Cases/subjects $(n)$ & $152 / 6856$ & & $1 / 6856$ & & /6857 & & /6856 & \\
\hline Median $(\mathrm{g})$ & 17.9 & & 36.5 & & $6 \cdot 4$ & & 34.9 & \\
\hline Age- and area-adjusted OR $\dagger$ & 1.00 (reference) & 1.08 & $0.86,1.35$ & $1 \cdot 12$ & $0.89,1.40$ & 1.38 & $1 \cdot 10,1 \cdot 72$ & 0.006 \\
\hline Multivariate-adjusted OR $\ddagger$ & 1.00 (reference) & 1.09 & $0.87,1.37$ & $1 \cdot 15$ & $0.91,1.46$ & 1.48 & $1.15,1.90$ & 0.003 \\
\hline Plus Fe-adjusted OR $\ddagger$ & 1.00 (reference) & 1.09 & $0.86,1.36$ & $1 \cdot 14$ & $0.90,1.44$ & 1.45 & $1 \cdot 13,1.86$ & 0.005 \\
\hline Plus saturated fat-adjusted OR & 1.00 (reference) & $1 \cdot 11$ & $0.88,1.40$ & $1 \cdot 19$ & $0.92,1.54$ & 1.58 & $1 \cdot 14,2 \cdot 20$ & 0.01 \\
\hline Unprocessed red meat & & & & & & & & \\
\hline Cases/subjects $(n)$ & $160 / 6856$ & & 7/6856 & & /6857 & & 7/6856 & \\
\hline Median $(\mathrm{g})$ & $15 \cdot 1$ & & 31.4 & & $9 \cdot 0$ & & $32 \cdot 7$ & \\
\hline Age- and area-adjusted OR $\dagger$ & 1.00 (reference) & 0.99 & $0.79,1.24$ & 1.00 & $0.80,1.25$ & 1.33 & $1.07,1.65$ & 0.01 \\
\hline Multivariate-adjusted OR $\ddagger$ & 1.00 (reference) & 0.99 & $0.79,1.24$ & 1.01 & $0.80,1.27$ & 1.42 & $1 \cdot 12,1 \cdot 81$ & 0.007 \\
\hline Plus Fe-adjusted OR $\ddagger$ & 1.00 (reference) & 0.99 & $0.79,1.24$ & 1.00 & $0.79,1.26$ & 1.39 & $1.09,1.77$ & 0.01 \\
\hline Plus saturated fat-adjusted OR $\ddagger$ & 1.00 (reference) & 1.01 & $0.80,1.27$ & 1.04 & $0.81,1.33$ & 1.51 & $1 \cdot 10,2 \cdot 06$ & 0.03 \\
\hline Processed red meat & & & & & & & & \\
\hline Cases/subjects $(n)$ & $167 / 6856$ & & 7/6856 & & $5 / 6857$ & & $2 / 6856$ & \\
\hline Median $(\mathrm{g})$ & 0.0 & & $2 \cdot 4$ & & 5.6 & & 14.6 & \\
\hline Age- and area-adjusted OR $\dagger$ & 1.00 (reference) & 1.02 & $0.82,1.28$ & 0.95 & $0.76,1.20$ & $1 \cdot 19$ & $0.94,1.51$ & 0.24 \\
\hline Multivariate-adjusted OR $\ddagger$ & 1.00 (reference) & 1.02 & $0.81,1.27$ & 0.94 & $0.75,1.19$ & $1 \cdot 19$ & $0.94,1.50$ & 0.27 \\
\hline Plus Fe-adjusted OR $\ddagger$ & 1.00 (reference) & 1.02 & $0.82,1 \cdot 27$ & 0.94 & $0.75,1.19$ & $1 \cdot 18$ & $0.93,1.49$ & 0.30 \\
\hline Plus saturated fat-adjusted OR & 1.00 (reference) & 1.01 & $0.81,1 \cdot 26$ & 0.93 & $0.74,1.17$ & $1 \cdot 15$ & $0.90,1.46$ & 0.43 \\
\hline Poultry & & & & & & & & \\
\hline Cases/subjects $(n)$ & $168 / 6856$ & & $9 / 6856$ & & $3 / 6857$ & & /6856 & \\
\hline Median $(\mathrm{g})$ & 0.0 & & $5 \cdot 1$ & & 9.6 & & 20.1 & \\
\hline Age- and area-adjusted OR $\dagger$ & 1.00 (reference) & 0.90 & $0.72,1 \cdot 12$ & 1.08 & $0.87,1.34$ & $1 \cdot 12$ & $0.91,1.39$ & $0 \cdot 12$ \\
\hline Multivariate-adjusted OR $\ddagger$ & 1.00 (reference) & 0.87 & $0.69,1.09$ & 1.02 & $0.82,1.27$ & 1.07 & $0.85,1.34$ & 0.38 \\
\hline Plus Fe-adjusted OR $\ddagger$ & 1.00 (reference) & 0.87 & $0.69,1.09$ & 1.01 & $0.81,1.26$ & 1.06 & $0.84,1.33$ & 0.41 \\
\hline Plus saturated fat-adjusted OR & 1.00 (reference) & 0.86 & $0.68,1.08$ & 0.99 & $0.80,1.24$ & 1.03 & $0.81,1.30$ & 0.59 \\
\hline Women & & & & & & & & \\
\hline Total meat & & & & & & & & \\
\hline Cases/subjects $(n)$ & $140 / 9106$ & & $7 / 9106$ & & /9106 & & /9106 & \\
\hline Median $(\mathrm{g})$ & 19.9 & & 39.4 & & $9 \cdot 2$ & & 94.4 & \\
\hline Age- and area-adjusted OR $\dagger$ & 1.00 (reference) & 0.94 & $0.73,1.19$ & 0.85 & $0.67,1 \cdot 10$ & 0.82 & $0.64,1.07$ & $0 \cdot 10$ \\
\hline Multivariate-adjusted OR $\ddagger$ & 1.00 (reference) & 0.97 & $0.76,1.25$ & 0.89 & $0.68,1.15$ & 0.82 & $0.62,1.09$ & 0.14 \\
\hline Plus Fe-adjusted OR $\ddagger$ & 1.00 (reference) & 0.97 & $0.76,1.25$ & 0.89 & $0.69,1.15$ & 0.83 & $0.62,1 \cdot 10$ & 0.15 \\
\hline Plus saturated fat-adjusted OR $\ddagger$ & 1.00 (reference) & 1.05 & $0.81,1.36$ & 1.03 & $0.77,1.37$ & $1 \cdot 11$ & $0.76,1.62$ & 0.68 \\
\hline Total red meat & & & & & & & & \\
\hline Cases/subjects $(n)$ & $143 / 9106$ & & $4 / 9106$ & & /9106 & & $2 / 9106$ & \\
\hline Median $(\mathrm{g})$ & $15 \cdot 2$ & & $32 \cdot 0$ & & 9.5 & & $32 \cdot 9$ & \\
\hline Age- and area-adjusted OR $\dagger$ & 1.00 (reference) & 0.90 & $0.70,1.14$ & 0.86 & $0.67,1.10$ & 0.77 & $0.60,1.01$ & 0.052 \\
\hline Multivariate-adjusted OR $\ddagger$ & 1.00 (reference) & 0.93 & $0.73,1.19$ & 0.89 & $0.69,1.15$ & 0.77 & $0.57,1.02$ & 0.08 \\
\hline Plus Fe-adjusted OR & 1.00 (reference) & 0.93 & $0.73,1 \cdot 20$ & $0 \cdot 89$ & $0.69,1 \cdot 16$ & $0 \cdot 77$ & $0.57,1.03$ & 0.08 \\
\hline Plus saturated fat-adjusted OR & 1.00 (reference) & 0.99 & $0.77,1.28$ & 1.01 & $0.76,1.35$ & 0.99 & $0.67,1.45$ & 0.99 \\
\hline Unprocessed red meat & & & & & & & & \\
\hline Cases/subjects $(n)$ & $137 / 9106$ & & 3/9106 & & /9106 & & 2/9106 & \\
\hline Median $(\mathrm{g})$ & $12 \cdot 3$ & & 26.9 & & $2 \cdot 4$ & & $72 \cdot 2$ & \\
\hline Age- and area-adjusted OR† & 1.00 (reference) & 1.00 & $0.79,1.27$ & $0 \cdot 87$ & $0.67,1.11$ & $0 \cdot 81$ & $0.62,1.05$ & 0.06 \\
\hline Multivariate-adjusted OR $\ddagger$ & 1.00 (reference) & 1.04 & $0.82,1.33$ & 0.91 & $0.71,1.18$ & 0.81 & $0.61,1.07$ & $0 \cdot 10$ \\
\hline Plus Fe-adjusted OR $\ddagger$ & 1.00 (reference) & 1.04 & $0.82,1.33$ & 0.92 & $0.71,1.19$ & 0.81 & $0.61,1.08$ & $0 \cdot 11$ \\
\hline Plus saturated fat-adjusted OR & 1.00 (reference) & $1 \cdot 11$ & $0.86,1.43$ & 1.03 & $0.76,1.37$ & 1.04 & $0.72,1.50$ & 0.90 \\
\hline Processed red meat & & & & & & & & \\
\hline Cases/subjects $(n)$ & $140 / 9106$ & & 5/9106 & & //9106 & & \$/9106 & \\
\hline Median $(g)$ & 0.0 & & $2 \cdot 4$ & & $5 \cdot 6$ & & 13.5 & \\
\hline Age- and area-adjusted OR† & 1.00 (reference) & 0.89 & $0.69,1.14$ & 0.98 & $0 \cdot 76,1 \cdot 25$ & 0.90 & $0 \cdot 70,1 \cdot 17$ & 0.61 \\
\hline Multivariate-adjusted OR $\ddagger$ & 1.00 (reference) & 0.94 & $0.73,1.22$ & 1.05 & $0.82,1.36$ & 0.96 & $0.73,1.26$ & 0.98 \\
\hline Plus Fe-adjusted OR $\ddagger$ & 1.00 (reference) & 0.94 & $0.73,1.22$ & 1.05 & $0.82,1.36$ & 0.96 & $0.73,1.27$ & 1.00 \\
\hline Plus saturated fat-adjusted OR $\ddagger$ & 1.00 (reference) & 0.97 & $0.75,1.26$ & $1 \cdot 11$ & $0.86,1.43$ & 1.05 & $0.79,1.40$ & 0.53 \\
\hline
\end{tabular}


Table 2. Continued

\begin{tabular}{|c|c|c|c|c|c|c|c|c|}
\hline & \multicolumn{7}{|c|}{ Quartile category } & \multirow[b]{3}{*}{$P$ for trend ${ }^{*}$} \\
\hline & \multirow{2}{*}{$\frac{\text { Q1 }}{\text { OR }}$} & \multicolumn{2}{|c|}{ Q2 } & \multicolumn{2}{|c|}{ Q3 } & \multicolumn{2}{|c|}{ Q4 } & \\
\hline & & OR & $95 \% \mathrm{Cl}$ & OR & $95 \% \mathrm{Cl}$ & OR & $95 \% \mathrm{Cl}$ & \\
\hline \multicolumn{9}{|l|}{ Poultry } \\
\hline Cases/subjects $(n)$ & $139 / 9106$ & \multicolumn{2}{|c|}{ 109/9106 } & \multicolumn{2}{|c|}{$125 / 9106$} & \multicolumn{2}{|c|}{$124 / 9106$} & \\
\hline Median $(\mathrm{g})$ & 0.0 & \multicolumn{2}{|c|}{4.5} & \multicolumn{2}{|c|}{$8 \cdot 6$} & \multicolumn{2}{|c|}{$17 \cdot 8$} & \\
\hline Age- and area-adjusted OR $†$ & 1.00 (reference) & 0.80 & $0.62,1.03$ & 0.93 & $0.73,1.19$ & 0.91 & $0.71,1 \cdot 17$ & 0.72 \\
\hline Multivariate-adjusted OR $\ddagger$ & 1.00 (reference) & 0.82 & $0.63,1.05$ & 0.94 & $0.73,1.21$ & 0.89 & $0.68,1.15$ & 0.56 \\
\hline Plus Fe-adjusted OR & 1.00 (reference) & 0.82 & $0.63,1.05$ & 0.94 & $0.73,1.21$ & 0.89 & $0.68,1.16$ & 0.58 \\
\hline Plus saturated fat-adjusted OR & 1.00 (reference) & 0.84 & $0.65,1.09$ & 0.99 & $0.77,1.28$ & 0.97 & $0.74,1.27$ & 0.94 \\
\hline
\end{tabular}

* Trend association was assessed by assigning the ordinal numbers $0-3$ to the four categories of each meat or specific groups of meat consumption

$\dagger$ Adjusted for age and public health centre area.

$\ddagger$ Additionally adjusted for BMI, smoking status, alcohol consumption, total physical activity, the history of hypertension, coffee consumption, the family history of diabetes, $\mathrm{Mg}$ intake, Ca intake, rice intake, fish intake, vegetable intake, soft drink consumption and energy intake.

the association with unprocessed red meat, is consistent with the result of two meta-analyses of data from prospective studies that were mainly conducted in Western populations (pooled hazard ratio $1 \cdot 21,95 \%$ CI $1 \cdot 07,1 \cdot 38^{(8)}$ and pooled hazard ratio $1 \cdot 19$, 95\% CI $1.04,1.37$ for $\left.100 \mathrm{~g} / \mathrm{d}^{(9)}\right)$. However, the absolute amount of total red meat intake in the present study population (median 45.6 and $40 \cdot 1 \mathrm{~g} / \mathrm{d}$ in men and women, respectively) was lower than that in the US population (mean $69.8 \mathrm{~g} / \mathrm{d}$ ) ${ }^{(34)}$, but was similar to that in the Chinese study population (median $42.6 \mathrm{~g} / \mathrm{d})^{(7)}$. Additionally, the type of unprocessed red meat consumed was considerably different among studies: pork intake was almost twice as high as beef intake in the USA ${ }^{(35)}$ and in the present study population, while in the Chinese study population, pork was consumed much more often than beef $(9: 1)^{(7)}$. Given that the Chinese study showed no increased risk of type 2 diabetes $^{(7)}$, beef intake might have a stronger influence on the risk of type 2 diabetes than pork intake. In fact, a randomised controlled trial has suggested that a regular intake of pork in place of other meats improves body composition ${ }^{(36)}$.

A positive association between processed meat and type 2 diabetes has been observed in three meta-analyses of cohort studies $^{(8-10)}$ and in prospective studies ${ }^{(4,5,14,15,20)}$ that were not included in those meta-analyses. The magnitudes of increases in risk associated with processed meat intake were greater than those associated with red meat intake ${ }^{(8-10)}$. For example, there were 41 and $21 \%$ increased risks for the highest $v$. lowest intake of processed meat and red meat, respectively $^{(8)}$. In the present study, however, men in the highest quartile of processed meat intake had a statistically insignificant $15 \%$ increased risk of type 2 diabetes compared with those in the lowest quartile. One explanation for this discrepancy is that processed meat consumption in Japan is much lower than in the USA (mean $12.4 \mathrm{~g} / \mathrm{d}$ in Japan and $23.2 \mathrm{~g} / \mathrm{d}$ in the USA $)^{(34,37)}$. In the present study population, the median in the highest quartile of processed red meat consumption was $13 \cdot 1 \mathrm{~g} / 8368 \mathrm{~kJ}(2000 \mathrm{kcal})$ per d among men and $14 \cdot 1 \mathrm{~g} / 8368 \mathrm{~kJ}$ ( $2000 \mathrm{kcal})$ per d among women. Similarly, in the Multiethnic Cohort in Hawaii, Japanese-American men and women in the highest category of processed red meat intake $(17 \cdot 1 \mathrm{~g} / 8368 \mathrm{~kJ}(2000 \mathrm{kcal})$ per $\mathrm{d}$ in men and $13.9 \mathrm{~g} / 8368 \mathrm{~kJ}(2000 \mathrm{kcal}) / \mathrm{d}$ in women) showed only modest increases in the risk of type 2 diabetes ( 24 and $23 \%$ for men and women, respectively) compared with those with the lowest category of meat intake ${ }^{(15)}$. Few Japanese are likely to consume processed red meat above the level that is apparently associated with the increased risk of type 2 diabetes.

Poultry intake was not appreciably associated with the risk of type 2 diabetes among either men or women in the present study. Previously, no association between poultry intake and the risk of type 2 diabetes has been consistently reported in men ${ }^{(4-6,14,15)}$. In women, however, data have been mixed $^{(4,7,14-16)}$, with two studies showing a decrease ${ }^{(7,16)}$, one study showing no association ${ }^{(15)}$ and two studies showing an increase in the risk of type 2 diabetes $^{(4,14)}$. Given that the majority of these studies did not detect an increased risk of type 2 diabetes associated with poultry intake, poultry intake may not impair glucose metabolism. There are some possible explanations. As poultry contains a lower amount of haem Fe than red meat, a higher intake of poultry may not significantly increase Fe storage, which has been linked to the risk of type 2 diabetes ${ }^{(38)}$. Moreover, PUFA in poultry can improve insulin sensitivity ${ }^{(39)}$.

In the present study, intakes of total meat, red meat and unprocessed red meat were associated with the risk of type 2 diabetes in men only. This could be ascribed, at least in part, to sex difference in Fe storage, which increases with the intake of Fe-rich foods including red meat. Circulating ferritin concentrations (a marker of Fe storage) in pre-menopausal women are much lower than those in $\operatorname{men}^{(38)}$, and showed no association with insulin resistance ${ }^{(40,41)}$. In postmenopausal women, whose blood ferritin levels are also lower than those in $\operatorname{men}^{(38)}$, studies on insulin resistance have yielded mixed results, with one US study reporting a positive association with ferritin concentrations ${ }^{(41)}$, whereas a Korean study reported no association ${ }^{(40)}$. In the present study, no increase in the risk of type 2 diabetes with a high intake of meat was observed among either pre- or postmenopausal women. The present finding may be supported by a Japanese study, in which serum ferritin concentrations were 
significantly associated with an insulin resistance marker in men but not in women ${ }^{(42)}$. For Asian women, meat intake may not increase Fe storage to the level above which glucose metabolism is impaired.

The mechanism underlying the association between meat intake and the risk of type 2 diabetes is unclear. Haem Fe in red meat has been suggested to play an unfavourable role in glucose metabolism ${ }^{(38)}$. Fe is a strong pro-oxidant and proinflammatory factor that catalyses several reactions leading to the formation of reactive oxygen species and resulting in elevated oxidative stress and inflammation ${ }^{(43)}$, which may decrease insulin sensitivity ${ }^{(38,44)}$. Increased Fe stores in the liver may induce insulin resistance by impeding the capacity for insulin extraction ${ }^{(38)}$. Fe impairs insulin action and interferes with glucose uptake in adipocytes ${ }^{(38)}$. In addition, increased muscle Fe stores enhance NEFA oxidation and cause them to interfere with glucose disposal ${ }^{(38)}$. Excess body Fe causes Fe deposition in pancreatic $\beta$-cells, resulting in impaired insulin secretion ${ }^{(38)}$. In epidemiological studies, intake of haem Fe has been consistently associated with an increased risk of type 2 diabetes ${ }^{(38)}$. In the present study, however, adjustment for Fe intake only slightly attenuated the association between total meat and total red meat and the risk of type 2 diabetes, indicating that mechanisms other than the latter may exist. Meat is also a major source of saturated fat, which is increased in cell membranes and leads to decreased membrane fluidity and decreased insulin receptor affinity $^{(45)}$. Saturated fat increases inflammatory responses and secondarily enhances the risk of type 2 diabetes ${ }^{(13)}$. However, the adjustment for saturated fat intake did not appreciably change the result in the present study. Alternatively, advanced glycation end products and heterocyclic amines, which are formed in meat through heating and processing ${ }^{(46,47)}$, have been shown to increase oxidative stress and inflammation, leading to the progression of insulin resistance in mice ${ }^{(48)}$. In a study of patients with type 2 diabetes, restriction of dietary advanced glycation end products improved insulin sensitivity ${ }^{(49)}$.

The major strengths of the present study include a large number of male and female participants, the populationbased prospective design, the use of a validated FFQ and adjustment for or stratification by potentially important confounding variables. Limitations of the present study also deserve mention. First, the diagnosis of type 2 diabetes was ascertained via self-report. We confirmed that $94 \%$ of selfreported diabetes cases were correctly documented in medical records in a sample of a validation study ${ }^{(33)}$, but undiagnosed cases may exist. It is unlikely, however, that the probability of under-diagnosis differs according to meat intake, and the use of self-reported data might not have substantially influenced the risk estimate. Second, we used only 5-year survey data for the assessment of dietary intake. Due to random variation, this would lead to a non-differential misclassification of meat intake and would thus distort the OR towards the null. In addition, one-time dietary measurement at baseline may not capture long-term intake, which is relevant to the development of type 2 diabetes. Repeated assessment of the diet over a long period of time before disease onset will probably provide a better estimate of exposure status. Third, although the validity of the FFQ for meat intake was relatively high ( $r$ 0.44-0.50), the measurement error in the FFQ might result in biased associations between meat intake and the risk of type 2 diabetes, which would drive the results towards the null. Fourth, we adjusted for dietary Fe intake but not for blood ferritin concentration, which is a better marker of body Fe status. Fifth, the follow-up period was relatively short ( 5 years). Finally, we could not rule out the possibility of unmeasured and residual confounding.

In conclusion, we found that total red meat and unprocessed red meat intakes were associated with the increased risk of type 2 diabetes after adjustment for other risk factors for type 2 diabetes among Japanese men, whose meat consumption is lower than that of Westerners. The present study adds to the evidence showing the adverse effect of high meat consumption on glucose metabolism.

\section{Acknowledgements}

The present study was supported by Grants-in-Aid for Cancer Research (19shi-2) and a Health Sciences Research Grant (Research on Comprehensive Research on Cardiovascular Diseases H19-016) from the Ministry of Health, Labour and Welfare of Japan.

The authors' contributions are as follows: S. T. was involved in the design of the study as the principal investigator; S. T. and M. I. conducted the survey; K. K., A. N., A. G., T. M., M. N., S. O., M. K. and Y. M. drafted the plan for the data analyses; K. K. conducted the data analyses; T. M. provided statistical expertise; K. K. drafted the manuscript; K. K. and T. M. had primary responsibility for the final content. All authors were involved in the interpretation of the results and the revision of the manuscript and approved the final version of the manuscript. None of the authors had a conflict of interest.

Members of the JPHC Study (principal investigator: S. T.) Group are as follows: S. T., M. I., T. Sobue and T. Hanaoka, National Cancer Center, Tokyo, Japan; J. Ogata, S. Baba, T. Mannami, A. Okayama and Y. Kokubo, National Cardiovascular Center, Osaka, Japan; K. Miyakawa, F. Saito, A. Koizumi, Y. Sano, I. Hashimoto, T. Ikuta and Y. Tanaba, Iwate Prefectural Ninohe Public Health Center, Iwate, Japan; Y. Miyajima, N. Suzuki, S. Nagasawa, Y. Furusugi and N. Nagai, Akita Prefectural Yokote Public Health Center, Akita, Japan; H. Sanada, Y. Hatayama, F. Kobayashi, H. Uchino, Y. Shirai, T. Kondo, R. Sasaki, Y. Watanabe, Y. Miyagawa and Y. Kobayashi, Nagano Prefectural Saku Public Health Center, Nagano, Japan; Y. Kishimoto, E. Takara, T. Fukuyama, M. Kinjo, M. Irei and H. Sakiyama, Okinawa Prefectural Chubu Public Health Center, Okinawa, Japan; K. Imoto, H. Yazawa, T. Seo, A. Seiko, F. Ito, F. Shoji and R. Saito, Katsushika Public Health Center, Tokyo, Japan; A. Murata, K. Minato, K. Motegi and T. Fujieda, Ibaraki Prefectural Mito Public Health Center, Ibaraki, Japan; K. Matsui, T. Abe, M. Katagiri and M. Suzuki, Niigata Prefectural Kashiwazaki and Nagaoka Public Health Center, Niigata, Japan; M. Doi, A. Terao, Y. Ishikawa and T. Tagami, Kochi Prefectural Chuo-higashi Public Health 
Center, Kochi, Japan; H. Sueta, H. Doi, M. Urata, N. Okamoto and F. Ide, Nagasaki Prefectural Kamigoto Public Health Center, Nagasaki, Japan; H. Sakiyama, N. Onga, H. Takaesu and M. Uehara, Okinawa Prefectural Miyako Public Health Center, Okinawa, Japan; F. Horii, I. Asano, H. Yamaguchi, K. Aoki, S. Maruyama, M. Ichii and M. Takano, Osaka Prefectural Suita Public Health Center, Osaka, Japan; Y. Tsubono, Tohoku University, Miyagi, Japan; K. Suzuki, Research Institute for Brain and Blood Vessels Akita, Akita, Japan; Y. Honda, K. Yamagishi, S. Sakurai and N. Tsuchiya, Tsukuba University, Ibaraki, Japan; M. Kabuto, National Institute for Environmental Studies, Ibaraki, Japan; M. Yamaguchi, Y. Matsumura, S. Sasaki and S. Watanabe, National Institute of Health and Nutrition, Tokyo, Japan; M. Akabane, Tokyo University of Agriculture, Tokyo, Japan; T. Kadowaki, Tokyo University, Tokyo, Japan; M. N. and T. M., National Center for Global Health and Medicine, Tokyo, Japan; Y. Kawaguchi, Tokyo Medical and Dental University, Tokyo, Japan; Y. Takashima and M. Yoshida, Kyorin University, Tokyo, Japan; K. Nakamura, Niigata University, Niigata, Japan; S. Matsushima and S. Natsukawa, Saku General Hospital, Nagano, Japan; H. Shimizu, Sakihae Institute, Gifu, Japan; H. Sugimura, Hamamatsu University, Shizuoka, Japan; S. Tominaga, Aichi Cancer Center Research Institute, Aichi, Japan; H. Iso, Osaka University, Osaka, Japan; M. Iida, W. Ajiki and A. Ioka, Osaka Medical Center for Cancer and CVD, Osaka, Japan; S. Sato, Chiba Prefectural Institute of Public Health, Chiba, Japan; E. Maruyama, Kobe University, Hyogo, Japan; M. Konishi, K. Okada and I. Saito, Ehime University, Ehime, Japan; N. Yasuda, Kochi University, Kochi, Japan; S. Kono, Kyushu University, Fukuoka, Japan.

\section{References}

1. Shaw JE, Sicree RA \& Zimmet PZ (2010) Global estimates of the prevalence of diabetes for 2010 and 2030. Diabetes Res Clin Pract 87, 4-14.

2. Iso H (2008) Changes in coronary heart disease risk among Japanese. Circulation 118, 2725-2729.

3. Nakanishi S, Okubo M, Yoneda M, et al. (2004) A comparison between Japanese-Americans living in Hawaii and Los Angeles and native Japanese: the impact of lifestyle westernization on diabetes mellitus. Biomed Pharmacother 58, 571-577.

4. Brand JS, van der Schouw YT, Onland-Moret NC, et al. (2012) Association between dietary meat consumption and incident type 2 diabetes: the EPIC-InterAct study. Diabetologia 56, 47-59.

5. Mannisto S, Kontto J, Kataja-Tuomola M, et al. (2010) High processed meat consumption is a risk factor of type 2 diabetes in the Alpha-Tocopherol, Beta-Carotene Cancer Prevention study. Br J Nutr 103, 1817-1822.

6. van Dam RM, Willett WC, Rimm EB, et al. (2002) Dietary fat and meat intake in relation to risk of type 2 diabetes in men. Diabetes Care 25, 417-424.

7. Villegas R, Shu XO, Gao YT, et al. (2006) The association of meat intake and the risk of type 2 diabetes may be modified by body weight. Int J Med Sci 3, 152-159.

8. Aune D, Ursin G \& Veierod MB (2009) Meat consumption and the risk of type 2 diabetes: a systematic review and meta-analysis of cohort studies. Diabetologia 52, 2277-2287.
9. Pan A (2011) Red meat consumption and risk of type 2 diabetes: 3 cohorts of US adults and an updated meta-analysis Am J Clin Nutr 94, 1088-1096.

10. Micha R (2010) Red and processed meat consumption and risk of incident coronary heart disease, stroke, and diabetes mellitus: a systematic review and meta-analysis. Circulation 121, 2271-2283.

11. Jiang R, Manson JE, Meigs JB, et al. (2004) Body iron stores in relation to risk of type 2 diabetes in apparently healthy women. JAMA 291, 711-717.

12. Tuomainen TP, Nyyssonen K, Salonen R, et al. (1997) Body iron stores are associated with serum insulin and blood glucose concentrations. Population study in 1,013 eastern Finnish men. Diabetes Care 20, 426-428.

13. Salas-Salvado J, Martinez-Gonzalez MA, Bullo M, et al. (2011) The role of diet in the prevention of type 2 diabetes. Nutr Metab Cardiovasc Dis 21, Suppl. 2, B32-B48.

14. Ericson U, Sonestedt E, Gullberg B, et al. (2013) High intakes of protein and processed meat associate with increased incidence of type 2 diabetes. Br J Nutr 109, 1143-1153.

15. Steinbrecher A, Erber E, Grandinetti A, et al. (2011) Meat consumption and risk of type 2 diabetes: the Multiethnic Cohort. Public Health Nutr 14, 568-574.

16. Schulze MB, Manson JE, Willett WC, et al. (2003) Processed meat intake and incidence of type 2 diabetes in younger and middle-aged women. Diabetologia 46, 1465-1473.

17. FAO (2012) FAO Statistical Yearbook World Food and Agriculture. Rome: FAO. http://www.fao.org/docrep/015/ i2490e/i2490e03c.pdf

18. Vang A, Singh PN, Lee JW, et al. (2008) Meats, processed meats, obesity, weight gain and occurrence of diabetes among adults: findings from Adventist Health Studies. Ann Nutr Metab 52, 96-104.

19. van Woudenbergh GJ (2012) Meat consumption and its association with C-reactive protein and incident type 2 diabetes: the Rotterdam Study. Diabetes Care 35, 1499-1505.

20. Lajous M, Tondeur L, Fagherazzi G, et al. (2012) Processed and unprocessed red meat consumption and incident type 2 diabetes among French women. Diabetes Care 35 $128-130$

21. Simmons RK, Harding AH, Wareham NJ, et al. (2007) Do simple questions about diet and physical activity help to identify those at risk of type 2 diabetes? Diabet Med $\mathbf{2 4}$, 830-835.

22. Fung TT, Schulze M, Manson JE, et al. (2004) Dietary patterns, meat intake, and the risk of type 2 diabetes in women. Arch Intern Med 164, 2235-2240.

23. Song Y, Manson JE, Buring JE, et al. (2004) A prospective study of red meat consumption and type 2 diabetes in middle-aged and elderly women: the women's health study. Diabetes Care 27, 2108-2115.

24. Sone H, Ito H, Ohashi Y, et al. (2003) Obesity and type 2 diabetes in Japanese patients. Lancet 361, 85 .

25. Jensen CC, Cnop M, Hull RL, et al. (2002) Beta-cell function is a major contributor to oral glucose tolerance in high-risk relatives of four ethnic groups in the U.S. Diabetes 51, $2170-2178$.

26. Szmuilowicz ED (2009) Influence of menopause on diabetes and diabetes risk. Nat Rev Endocrinol 5, 553-558.

27. Tsugane S \& Sobue T (2001) Baseline survey of JPHC study - design and participation rate. Japan Public Health Centerbased Prospective Study on Cancer and Cardiovascular Diseases. J Epidemiol 11, S24-S29.

28. Sasaki S, Kobayashi M, Ishihara J, et al. (2003) Self-administered food frequency questionnaire used in the 5-year follow-up survey of the JPHC Study: questionnaire structure, 
computation algorithms, and area-based mean intake. J Epidemiol 13, S13-S22.

29. Science and Technology Agency (2005) Standard Tables of Food Composition in Japan, 5 th revised and enlarged edition. Tokyo: Printing Bureau of the Ministry of Finance.

30. Ishihara J, Sobue T, Yamamoto S, et al. (2003) Validity and reproducibility of a self-administered food frequency questionnaire in the JPHC Study Cohort II: study design, participant profile and results in comparison with Cohort I. J Epidemiol 13, S134-S147.

31. Sasaki S, Ishihara J, Tsugane S, et al. (2003) Reproducibility of a self-administered food frequency questionnaire used in the 5-year follow-up survey of the JPHC Study Cohort I to assess food and nutrient intake. $J$ Epidemiol 13, S115-S124.

32. Sasaki S, Kobayashi M, Tsugane S, et al. (2003) Validity of a self-administered food frequency questionnaire used in the 5-year follow-up survey of the JPHC Study Cohort I: comparison with dietary records for food groups. J Epidemiol 13, S57-S63.

33. Kato M, Noda M, Inoue M, et al. (2009) Psychological factors, coffee and risk of diabetes mellitus among middle-aged Japanese: a population-based prospective study in the JPHC study cohort. Endocr J 56, 459-468.

34. Daniel CR, Cross AJ, Koebnick C, et al. (2011) Trends in meat consumption in the USA. Public Health Nutr 14, 575-583.

35. Bachman JL, Reedy J, Subar AF, et al. (2008) Sources of food group intakes among the US population, 2001-2002. J Am Diet Assoc 108, 804-814.

36. Murphy KJ, Thomson RL, Coates AM, et al. (2012) Effects of eating fresh lean pork on cardiometabolic health parameters. Nutrients 4, 711-723.

37. Kenko Eiyo Joho K (2010) The National Health and Nutrition Survey in Japan, 2007. Tokyo: Daiichi-shuppan.

38. Rajpathak SN, Crandall JP, Wylie-Rosett J, et al. (2009) The role of iron in type 2 diabetes in humans. Biochim Biophys Acta 1790, 671-681.
39. Lichtenstein AH (2000) Relationship of dietary fat to glucose metabolism. Atherosclerosis 150, 227-243.

40. Lee BK, Kim Y \& Kim YI (2011) Association of serum ferritin with metabolic syndrome and diabetes mellitus in the South Korean general population according to the Korean National Health and Nutrition Examination Survey 2008. Metabolism 60, 1416-1424.

41. Jehn M, Clark JM \& Guallar E (2004) Serum ferritin and risk of the metabolic syndrome in U.S. adults. Diabetes Care 27, $2422-2428$.

42. Pham NM, Nanri A, Yi S, et al. (2013) Serum ferritin is associated with markers of insulin resistance in Japanese men but not in women. Metabolism 62, 561-567.

43. Wagener FADTG (2003) Different faces of the heme-heme oxygenase system in inflammation. Pharmacol Rev 55, $551-571$.

44. Haffner SM (2006) The metabolic syndrome: inflammation, diabetes mellitus, and cardiovascular disease. Am J Cardiol 97, 3A-11A.

45. Ginsberg BH, Brown TJ, Simon I, et al. (1981) Effect of the membrane lipid environment on the properties of insulin receptors. Diabetes 30, 773-780.

46. Sinha R, Rothman N, Salmon CP, et al. (1998) Heterocyclic amine content in beef cooked by different methods to varying degrees of doneness and gravy made from meat drippings. Food Chem Toxicol 36, 279-287.

47. Hofmann SM, Dong HJ, Li Z, et al. (2002) Improved insulin sensitivity is associated with restricted intake of dietary glycoxidation products in the $\mathrm{db} / \mathrm{db}$ mouse. Diabetes $\mathbf{5 1}$, 2082-2089.

48. Cai W, He JC, Zhu L, et al. (2008) Oral glycotoxins determine the effects of calorie restriction on oxidant stress, age-related diseases, and lifespan. Am J Pathology 173, 327-336.

49. Uribarri J, Cai W, Ramdas M, et al. (2011) Restriction of advanced glycation end products improves insulin resistance in human type 2 diabetes: potential role of AGER1 and SIRT1. Diabetes Care 34, 1610-1616. 